\section{Aortic valve endocarditis with Erysipelothrix rhusiopathiae: A rare zoonosis}

\author{
Jenny Jónsdóttir Nielsen, ${ }^{1,2}$ \\ Bjørn Blomberg, ${ }^{3}$ Shahin Gaïni, ${ }^{2,4,5}$ \\ Steinar Lundemoen ${ }^{6}$ \\ ${ }^{1}$ Department of Infectious Diseases, \\ Odense University Hospital, Denmark; \\ ${ }^{2}$ Medical Department, Infectious \\ Diseases Division, National Hospital \\ Faroe Islands, Tórshavn, Faroe Islands; \\ ${ }^{3}$ Department of Thoracic Surgery, \\ Haukeland University Hospital, Bergen, \\ Norway; ${ }^{4}$ Infectious Diseases Research \\ Unit, Odense University Hospital and \\ University of Southern Denmark, \\ Denmark; ${ }^{5}$ Centre of Health Research \\ and Department of Science and \\ Technology, University of the Faroe \\ Islands, Tórshavn, Faroe Islands; \\ ${ }^{6}$ National Centre for Tropical Infectious \\ Diseases, Haukeland University \\ Hospital, Bergen, Norway
}

\begin{abstract}
Erysipelothrix rhusiopathiae has an economic impact in animal husbandry by causing infection in swine, sheep and poultry. E. rhusiopathiae is present in the surface mucoid slime on fish, although fishes do not seem to be affected. Humans can get infected, maost often through occupational exposure and may suffer typical erysipeloid infection on exposed skin such as on hands and fingers, or deeper skin infections, and sometimes sepsis and endocarditis, associated with high case-fatality rate. We describe a case of aortic valve endocarditis caused by E. rhusiopathiae in a 59-year-old man who enjoyed fishing in his spare time.
\end{abstract}

\section{Introduction}

Infection by E. rhusiopathiae, which is called red-disease by veterinarians, ${ }^{1}$ is a zoonosis that presents in three forms in humans: as a local skin lesion called erysipeloid; as a diffuse cutaneous infection with systemic symptoms; or as septicemia, with or without endocarditis. Infections are frequently acquired through occupational exposure. $^{2,3}$ E. rhusiopathiae infection is uncommon in humans and is a very rare cause of aortic valve endocarditis. ${ }^{2}$ We present here the first known case of aortic valve endocarditis caused by an E. rhusiopathiae infection in the Faroe Islands.

\section{Case Report}

A 59-year-old man from the Faroe Islands presented in August 2015 with malaise, double vision and light ataxia at the neurology department of a regional hospital in Norway. Except for prior surgery for varicose veins, hernia and kidney stones, he considered himself to be in good health. He was married with three children and worked on a supply ship offshore of Norway. He reported abstinence from alcohol on the 3-week work shifts, but daily alcohol consumption when on leave (3 weeks). He was an active smoker with a history of approximately 40 pack-years.

Ten days prior to admission, he sustained a fall injury, followed by nose bleeding and a feeling of tiredness that lasted for several days. He did not have any headache and had not noticed whether he had hit his head. Four days later he felt sufficiently well to start working on the supply ship in Norway as planned. Three days prior to hospital admission, the patient experienced a tingling sensation in all fingers of his right hand that was succeeded by pain in the ulnar fingers of his left hand, which was greatest in the fifth finger. The following day the patient suddenly noticed binocular double vision and an unsteady gait. He reported feeling weakness and fatigue. He had a temperature of $39^{\circ} \mathrm{C}$ and dark, foul smelling urine, which prompted the chief commander on board the supply ship to start treatment with oral ciprofloxacin for a suspected urinary tract infection.

During examination at the neurology department, the patient was awake, conscious and oriented. His temperature was $37.3^{\circ} \mathrm{C}$, blood pressure $130 / 62 \mathrm{mmHg}$, heart rate 73 beats/minute, respiratory rate 14 breaths/minute, and oxygen saturation $96 \%$ without supplementation. He was not septic. Cardiac auscultation and ECG were normal. Neurological examination revealed upbeat nystagmus, intermittent double vision and ataxic gait. The fifth finger of the left hand was red and sore. Blood tests showed minimal anemia, thrombocytopenia $82 \times 10^{9} / \mathrm{L}, \mathrm{C}$ reactive protein (CRP) 34 $\mathrm{mg} / \mathrm{mL}$, hematuria (+3 urine dip test) and proteinuria (+2 urine dip test). Due to suspicion of central cerebral pathology, the patient was transferred to the stroke ward.

The magnetic resonance imaging (MRI) of the brain revealed a small subdural hematoma in the left cerebral hemisphere, hemorrhage in the right thalamus and multiple small lesions in both hemispheres. The small lesions were interpreted as new and older micro bleedings. MRI angiography was normal. Systemic illnesses including cancer and hematological diseases were
Correspondence: Jenny Jónsdóttir Nielsen, Department Infectious Diseases, Dept. Q, Odense University Hospital, J.B.Winsløwsvej 4, DK-5000 Odense C, Denmark.

Tel.: +45.66113333

E-mail: jenny.jonsdottir.nielsen@rsyd.dk

Key words: Erysipelothrix rhusiopathiae; endocarditis; zoonosis.

Contributions: JJN wrote the manuscript draft; SL and SG treated the patient; SG, BB and SL contributed to the manuscript preparation.

Conflict of interest: the authors declare no potential conflict of interest.

Funding: none

Received for publication: 4 June 2018.

Revision received: 11 July 2018.

Accepted for publication: 11 July 2018.

This work is licensed under a Creative Commons Attribution-NonCommercial 4.0 International License (CC BY-NC 4.0).

(C)Copyright J.J. Nielsen et al., 2018

Licensee PAGEPress, Italy

Infectious Disease Reports 2018; 10:7770

doi:10.4081/idr.2018.7770

considered based on the findings of the cerebral MRI, nosebleed, hematuria and malaise.

The patient had a temporary elevation in CRP and a sedimentation rate of 89-95 units. Kidney parameters and biochemical cancer markers were normal. Chest X-ray showed signs of pulmonary edema notably in the right hilum, and hand X-ray was normal. Transthoracic echocardiography (TTE) revealed aortic valve endocarditis with a main lesion of $1.2 \times 1.1 \mathrm{~cm}$, perforation of the non-coronal cusp, and severe heart insufficiency. No vegetation was found elsewhere. Blood was drawn for culture and treatment with intravenous benzylpenicillin 3 grams 4 times daily combined with intravenous gentamicin $500 \mathrm{mg}$ once daily was initiated. The patient was diagnosed with endocarditis with septic emboli to the brain and possibly to the kidneys and joints.

Eleven days after initial admission, $E$. rhusiopathiae was identified by MALDITOF in two out of four blood culture bottles (BacT/ALERT from Biomerieux). Antibiotic susceptibility test (Liofilchem ${ }^{\circledR}$ MIC Test Strip) showed the following minimum inhibitory concentrations (MIC): penicillin $0.03 \mu \mathrm{g} / \mathrm{mL}$, clindamycin 0.12 $\mu \mathrm{g} / \mathrm{mL}$, ceftriaxone $0.06 \mu \mathrm{g} / \mathrm{mL}$, ciprofloxacin $0.12 \mu \mathrm{g} / \mathrm{mL}$, vancomycin 64 $\mu \mathrm{g} / \mathrm{mL}$, daptomycin $0.5 \mu \mathrm{g} / \mathrm{mL}$, gentamicin-resistant. MIC values were interpreted 
based on the CLSI and EUCAST interpretative criteria of MIC break points, and the $E$. rhusiopathiae was sensitive to penicillin, clindamycin, ceftriaxone, ciprofloxacin and daptomycin and resistant to gentamicin and vancomycin. Based on these results the antibiotic treatment was changed to monotherapy with intravenous benzylpenicillin.

A TEE (transesophageal echocardiography) revealed a growing vegetation and increasing aortic valve insufficiency and the patient was moved to the cardiac department of Haukeland University Hospital 14 days after initial admission, in preparation for possible heart surgery. Benzylpenicillin dose was increased to 2.4 grams 6 times daily. No new neurological abnormalities or arrhythmias were evident and the patient was respiratory and circulatory stable. He had a weak systolic murmur with punctum maximum over right IC2-3, the fifth toe on the left foot was red and warm, and the left hand appeared normal but movement was reduced in the distal interphalangeal-joint of the fifth finger. A new MRI revealed new septic emboli in the right cerebral hemisphere (Figure 1).

TEE on day 16 showed aortic valve endocarditis with perforation of the noncoronal cusp and fluttering vegetation from the cusp $(1.4 \times 0.9 \mathrm{~cm})$. A computerized tomography (CT) scan of the heart showed normal vessels.

The presence of several dental abscesses and chronic marginal periodontitis prompted the need for oral surgery. Teeth number 16, 17, 25 and 27 were extracted and an alveoantral fistula was closed.

Three weeks after initial presentation, a new diastolic murmur was registered. TEE showed the same perforation of the noncoronal cusp and severe aortic insufficiency. A $1.1 \times 0.8 \mathrm{~cm}$ fluttering vegetation was present on the perforated cusp and a phlegmonous swelling in the aortic root and mild mitral valve insufficiency was discovered.

On day 29 after initial admission, the patient received a PERIMOUNT $(25 \mathrm{~mm}$, model 2900) biological aortic valve because of the increasing leak. The patient opted for the biological valve as he did not want to be bound to vitamin-K antagonistic therapy, considering his offshore job.

During surgery the native aortic valve was found carrying vegetations on all three cusps, the non-coronal cusp was perforated and the right coronary cusp was nearly perforated. There was no abscess. The surgery was successful, but in the postoperative period the patient experienced some problems with arrhythmias and an episode of Pseudomonas urosepsis. Urosepsis was initially treated with intravenous ceftriaxone 2 grams daily and after three days changed to intravenous ciprofloxacin $400 \mathrm{mg}$ twice a day for ten days.

Microbiological investigation of the removed valve did not reveal bacteria growth by conventional culture methods. However, broad range 16s RNA PCR for bacterial DNA followed by sequencing confirmed the presence of E. rhusiopathiae DNA on the valve, as suspected after the blood culture results.

Fourteen days after surgery, the patient had good valve function measured by echocardiography and was discharged from the Haukeland University hospital. The patient was transferred to the National Hospital Faroe Islands to complete the sixweeks course of intravenous treatment with benzylpenicillin 2.4 grams 6 times daily that had been initiated on the day E. rhusiopathiae was first identified in blood culture.

The patient recovered fully and returned to his job in January 2016. He was followed up at the Cardiac and Infectious Diseases Outpatient Clinics at the National Hospital Faroe Islands. TTE in September 2016 showed a left ventricular ejection fraction of $50-55 \%$ and a well-functioning prosthetic aortic valve.

Written consent for a case report publication was obtained from the patient.

\section{Discussion and Conclusions}

E. rhusiopathiae are gram-positive rodshaped bacteria (Figure 2), living in the gastrointestinal tract and lymphoid tissues of domestic and wild animals including pigs, sheep and poultry, as well as in the superficial mucoid slime on fish. The bacteria can also be found in the environment where they can survive for a long time even if they do not form spores. ${ }^{3}$ Mosquitoes have been identified as temporary vectors. ${ }^{2,3}$

Human infection commonly arises through skin lesions resulting in a focal, painful but self-limiting erysipeloid. Occasionally diffuse cellulitis is observed and in rare cases E. rhusiopathiae can cause sepsis, endocarditis, arthritis and meningitis. $^{2}$

E. rhusiopathiae can cause disease in pigs and poultry although it has been assessed that a large proportion of the animals are asymptomatic carriers. ${ }^{4,5}$ The clinical manifestations of disease in pigs are the same as in humans. ${ }^{4,5}$ E. rhusiopathiae also cause severe infections in turkeys and is an emerging problem in hen-farming. ${ }^{4}$ Vaccines have been developed for pigs and sheep, but not for poultry. ${ }^{4}$ In Denmark ${ }^{1}$ and in Germany ${ }^{4}$ barn sows are vaccinated reg- ularly, which has reduced the farmers' economic losses. E. rhusiopathiae does not cause disease in fish but it survives on the exterior mucoid slime, from where the bacteria can be transferred to humans. 3,6

Infection with E. rhusiopathiae is an occupational hazard for people working with animal husbandry and fishing and involves exposure to infected animals.,7 People at greatest risk of getting infected with E. rhusiopathiae are 40/60-year-old men. Other risk factors include excessive alcohol consumption, malnutrition and dia-

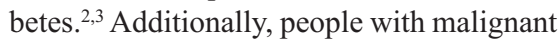
diseases, tuberculosis, liver cirrhosis or people treated with cytostatics or corticoids are at higher risk of life-threatening E. rhu-

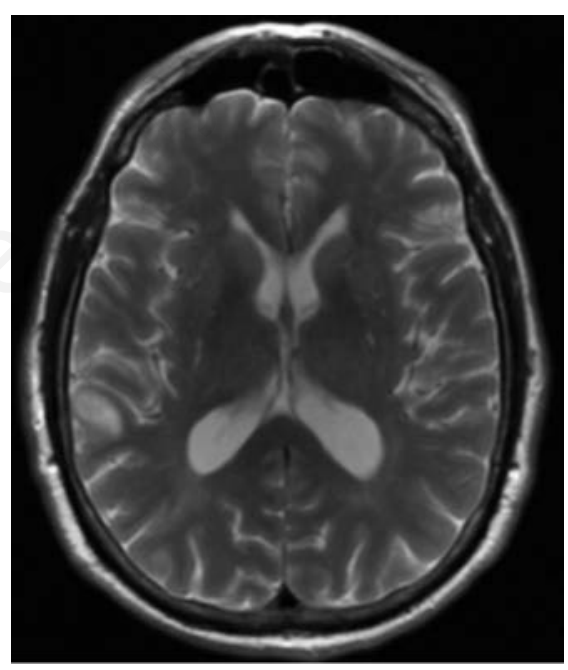

Figure 1. MRI of the brain showing septic emboli in the right cerebral hemisphere (MRT2TseTra2).

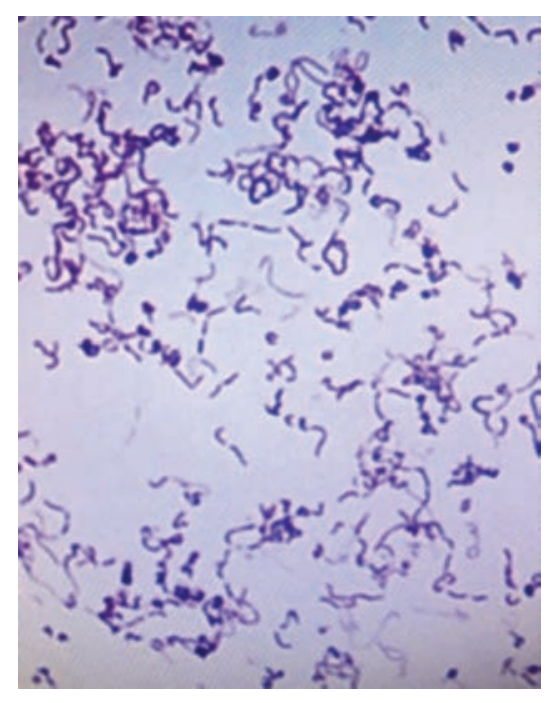

Figure 2. Microscopic findings of $E$. rhusiopathiae. Gram positive, rod-shaped bacteria. 
siopathiae infections. ${ }^{2}$

Endocarditis caused by E. rhusiopathiae has a $35-40 \%$ mortality rate which is higher than endocarditis caused by most other bacterial etiologies. , $^{2,3}$,

As mentioned earlier, infections with $E$. rhusiopathiae are very rare, or seldom reported, in humans. A survey conducted in the UK showed that the annual registered number of human cases of bacteremia with E. rhusiopathiae in 2010-2014 was 7, 3, 10, 2 and 2 , respectively. ${ }^{8}$

In a publication in 2015 Ping Hua et al. ${ }^{2}$ state that since 1912, 60 cases of endocarditis with E. rhusiopathiae have been registered, of which less than 10 included aortic valve endocarditis. A broad search on Pubmed [E. rhusiopathiae AND endocarditis] resulted in one publication of human aortic valve endocarditis and non-specified endocarditis caused by E. rhusiopathiae after the mentioned publication from 2015 . $^{7}$

To our knowledge, our case is the first of human endocarditis caused by E. rhusiopathiae in the fish- and sheep-farming community of the Faroe Islands. Iceland similarly has a great fish and sheep industry with no known cases of endocarditis caused by E. rhusiopathiae (Personal communication: Karl G. Kristinsson, MD).

Our patient was hobby fishing in July 2015. He had a dog, but had no contact with other animals. Furthermore, he cannot recall having a wound in July 2015. However, considering his reported fishing in July 2015, he most likely contracted the bacteria when gutting the fish he caught. The risk factors of our patient were gender, age and a moderate to high alcohol consumption, which is a known risk factor for E. rhusiopathiae infection.

E. rhusiopathiae is usually sensitive to penicillin, cephalosporins and clindamycin. ${ }^{3,9}$ Penicillin is usually the drug of choice and cephalosporins in individuals allergic to penicillin. ${ }^{3}$

Most veterinarians are aware of E. rhusiopathiae, ${ }^{1}$ and pig farmers fear it and vac- cinate their animals against it due to the economic impact of this disease.

Even though Denmark has a very large pig farming industry, no case of E. rhusiopathiae endocarditis has been registered in the East Denmark Endocarditis Database, which includes more than 1000 endocarditis cases in the period 2002-2012 (personal communication: Niels Eske Bruun, MD).

In the Faroe Islands, farmers and others who handle sheep are aware of the risk of developing erysipeloid and take precautions to avoid it. Infected wounds are commonly treated with phenoxymethylpenicillin empirically. Faroese sheep are not vaccinated against E. rhusiopathiae. In the Faroese fishing industry, E. rhusiopathiae or erysipeloid is not well known and is not a common concern among fishermen. To our knowledge, E. rhusiopathiae is not of major clinical importance among Faroese and Icelandic fishermen (Personal communication: Shahin Gaïni, MD; Karl G. Kristinsson, MD).

Good hygiene is assumed to be the best protection from infection, in particular frequent wash with disinfecting soap when handling a potential source of infection, the use of gloves, and quick treatment of wounds. Another suggested protective factor is to keep up good health and immune status of the people taking care of the animals. ${ }^{4}$ Primary prevention in animals can contribute to controlling the disease.

Invasive infections such as endocarditis caused by E. rhusiopathiae is potentially life-threatening, and is associated with worse outcome than endocarditis caused by other more common organisms. Clinicians working with exposed populations, such as fishing communities and animal husbandry workers, should be aware of this microbe and its clinical manifestations.

\section{References}

1. Haugegaard J. Rødsyge - stadig aktuel. Dansk Veterinærtidsskrift 2005;14:9-11.
2. Hua P, Liu J, Tao J, et al. Erysipelothrix rhusiopathiae-induced aortic valve endocarditis: case report and literature review. Int J Clin Exp Med 2015;8:7306.

3. Brooke CJ, Riley TV. Erysipelothrix rhusiopathiae: bacteriology, epidemiology and clinical manifestations af an occupational pathogen. Med Microbiol 1999;48:789-99.

4. Janßen T, Voss M, Küh M, et al. A combinational approach of multilocus sequence typing and other molecular typing methods in unraveling the epidemiology of Erysipelothrix rhusiopathiae strains from poultry and mammals. Vet Res 2015;46:84.

5. Ding Y, Zhu D, Zang J, et al. Virulence determinants, antimicrobial susceptibility, and molecular profiles of Erysipelothrix rhusiopathiae strains isolated in China. Emerg Microbes Infect 2015;4:e69.

6. Yamamoto Y, Shioshita K, Takazono T, et al. An autopsy case of Erysipelothrix rhusiopathiae endocarditis. Intern Med 2008;47:1437-40.

7. Vázquez L, De Los Santos C, Cichero $\mathrm{M}$, et al. R. Infective endocarditis caused by Erysipelothrix rhusiopathie. Report of one case. Rev Med Chil 2015;143:1598-600.

8. UK Government, Public Health England. Health Protection Report, Vol. 9, No. 45, 2015. Available from: http://webarchive.nationalarchives.gov. uk/20161129091229/https:/www.gov.u k/government/uploads/system/uploads/ attachment_data/file/487560/hpr4515 ncmmns.pdf

9. Principe L, Bracco S, Mauri C, et al. Erysipelothrix rhusiopathiae bacteremia without endocarditis: Rapid identification from positive blood culture by MALDI-TOF mass spectrometry. A case report and literature review. Infect Dis Rep 2016;8:6368. 